\title{
Hitting probability for anomalous diffusion processes
}

\author{
Satya N. Majumdar and Alberto Rosso \\ CNRS - Université Paris-Sud, LPTMS, UMR8626 - Bât. 100, 91405 Orsay Cedex, France \\ Andrea Zoid* \\ CEA/Saclay, DEN/DM2S/SERMA/LTSD, Bât. 454, 91191 Gif-sur-Yvette Cedex, France
}

\begin{abstract}
We present the universal features of the hitting probability $Q(x, L)$, the probability that a generic stochastic process starting at $x$ and evolving in a box $[0, L]$ hits the upper boundary $L$ before hitting the lower boundary at 0 . For a generic self-affine process (describing, for instance, the polymer translocation through a nanopore) we show that $Q(x, L)=Q(x / L)$ and the scaling function $Q(z) \sim z^{\phi}$ as $z \rightarrow 0$ with $\phi=\theta / H$ where $H$ and $\theta$ are respectively the Hurst exponent and the persistence exponent of the process. This result is verified in several exact calculations including when the process represents the position of a particle diffusing in a disordered potential. We also provide numerical supports for our analytical results.
\end{abstract}

The transfer of DNA, RNA and proteins through cell membranes is key to understanding several biological processes [1]. The transport of polymer molecules across nanopores is also relevant in many chemical and industrial applications [2]. A fundamental question concerns whether a polymer, once penetrated into the pore, will eventually complete its transit. The answer is naturally formulated in terms of the translocation coordinate $X(t)$, namely the length of the translocated portion of the polymer at time $t$ 3, 4, 5, 6. In absence of driving forces, the polymer dynamics is governed by thermal fluctuations. In this case, the traslocation coordinate can be expressed as a stochastic process $X(t)$ that evolves in a box of size $L$ ( $L$ being the polymer length), starting from some initial value $X(0)=x, 0<x<L$, and terminated upon touching either boundary for the first time (Fig. 1 left). It has been shown that excluded volume effects hinder the polymer dynamics, so that the process $X(t)$ actually undergoes subdiffusion [5, 7. We define the hitting probability $Q(x, L)$ as the probability of exiting the domain through the boundary at $L$, which corresponds to the polymer completing the translocation.

More generally, the hitting probability $Q(x, L)$ of a particle undergoing anomalous (i.e., non-Brownian) diffusion is key to understanding a variety of phenomena, such as the classical gambler's ruin problem in finance and risk management $[8,9$, the transport of charge carriers in conductors with disordered impurities [10] and the breakthrough of chemical species in heterogeneous porous media for contaminated sites remediation [11, only to name a few. For ordinary Brownian diffusion, the hitting probability $Q(x, L)=x / L$ is easy to compute $[8,9$. The goal of this Letter is to study $Q(x, L)$ for generic self-affine stochastic processes, thus going beyond the Brownian world.

Since the only length scale in the problem is $L$, evidently $Q(x, L)$ is a function of only the scaled variable $x / L: Q(x, L)=Q(x / L=z)$. For a Brownian motion, $Q(z)=z$, a simple linear function. For a generic stochastic process $X(t), Q(z)$ is non trivial (see for example
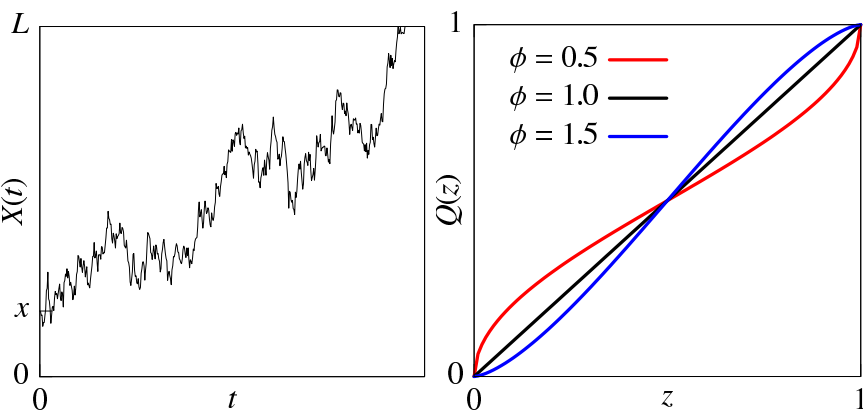

FIG. 1: Left. The evolution of a stochastic process initiated at $X(0)=x$ and terminated upon exiting from the box of size $L$. Right. The function $Q(z)$ as given by Eq. (8) for different values of the exponent $\phi$.

Fig. 1 right). The central aim of this Letter is to determine the universal features associated with $Q(z)$ in the two following cases: symmetric self-affine processes, characterized by a power-law scaling $X(t) \sim t^{H}$, with Hurst exponent $H>0$; and a single particle diffusing in a disordered potential $V(X)$. The translocation process belongs to the former, whereas transport in quenched disorder to the latter.

It is useful to summarize our main results which are threefold: $i$ ) For self-affine processes, we show that generically $Q(z) \sim z^{\phi}$ for small $z$, where $\phi=\theta / H$, and $\theta$ is the so-called persistence exponent [12] of the same process in a semi-infinite geometry. ii) For a particle diffusing in a disordered potential $V(X)$, we provide an exact formula for $Q(x, L)$ valid for arbitrary $V(X)$ which incidentally also allows us to compute the persistence exponent of a particle diffusing in a self-affine disordered potential. iii) The function $Q(z)$ is explicitly known for some anomalous diffusion processes. Amazingly, we find that these apparently different-looking formulae can be cast in the same super-universal form, when expressed in terms of the exponent $\phi$. This naturally raises the question: how generic is this super-universality? We provide numerical evidences that indeed in some cases the super- 

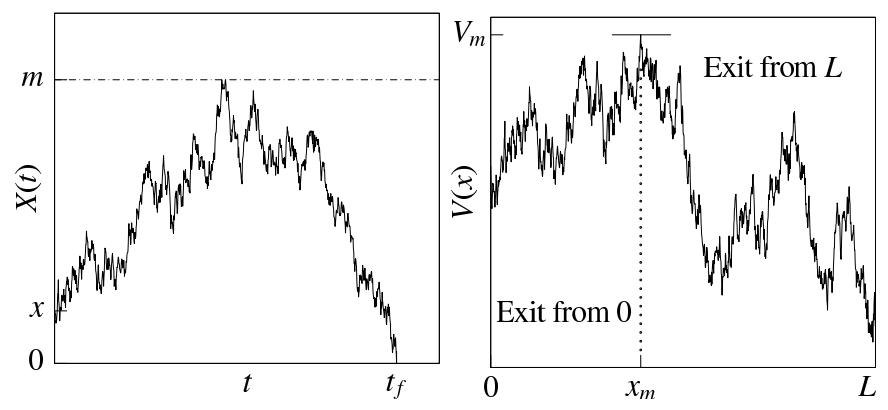

FIG. 2: Left. A stochastic process starting at $x$ leaves the positive half axis for the first time at $t_{f} ; m$ denotes its maximum till $t_{f}$. Right. A self-affine disordered potential with maximum at $x_{m}$ : when $L$ is large, the diffusing particle, starting at $0<x<L$, exits the box through 0 for $x<x_{m}$ and through $L$ for $x>x_{m}$.

universality is violated and we discuss its limit of validity.

Self-affine processes. To compute $Q(x, L)$ in a box geometry, it is useful first to relate it to another quantity associated with the same process $X(t)$, but now in a semi-infinite geometry $[0, \infty]$. Consider a process $X(t)$ in $[0, \infty]$, starting at $x$ and absorbed at the origin for the first time at $t_{f}-$ the first-passage time. Let $m$ denote the maximum of this process till $t_{f}$ (see Fig. 2left). Then, it is clear that $1-Q(x, L)$, the probability that the particle exits the box through the origin (and not through $L$ ), is precisely equal to the probability that the maximum $m$ of the process in $[0, \infty]$ till $t_{f}$ stays below $L$, i.e., the cumulative distribution of $m$, $\operatorname{Prob}[m \leq L \mid x]$, in the semi-infinite geometry. The distribution of $m$ is, in turn, related to the distribution of the first-passage time $t_{f}$. Let $q(x, T)=\operatorname{Prob}\left[t_{f} \geq T \mid x\right]$ denote the cumulative probability of $t_{f}$, which is also the survival probability of the particle starting at $x$ in the semi-infinite geometry. One knows that for generic self-affine processes $q(x, T)=q\left(x / T^{H}\right)$. For large $T, q(x, T) \sim T^{-\theta}$, where $\theta$ is the persistence exponent of the process [12]. This implies the scaling function $q(y) \sim y^{\theta / H}$ for small $y$ [7. Noting that $m \sim t_{f}^{H}$ for self-affine processes, it follows that $Q(x, L)=1-\operatorname{Prob}[m \leq L \mid x]=\operatorname{Prob}[m \geq$ $L \mid x] \approx \operatorname{Prob}\left[t_{f} \geq L^{1 / H} \mid x\right]=q[x / L]$. This demonstrates the scaling behavior anticipated before, namely, $Q(x, L)=Q(x / L)$, where $Q(z)=q(z)$. Moreover, since $q(y) \sim y^{\theta / H}$ for small $y$, we get $Q(z) \sim z^{\phi}$ for small $z$, with $\phi=\theta / H$. For example, for Brownian motion $H=1 / 2$ and $\theta=1 / 2$, hence $\phi=1$, in accordance with the exact result $Q(z)=z$. For the subclass of self-affine processes with stationary increments, the same exponent $\phi$ happens to describe the vanishing of the probability density close to an absorbing boundary [7].

Our general prediction $Q(z) \sim z^{\phi}$ for small $z$ can be verified explicitly for some self-affine processes where $Q(z)$ can be computed exactly, as discussed later. Moreover, we have numerically verified that this conjecture

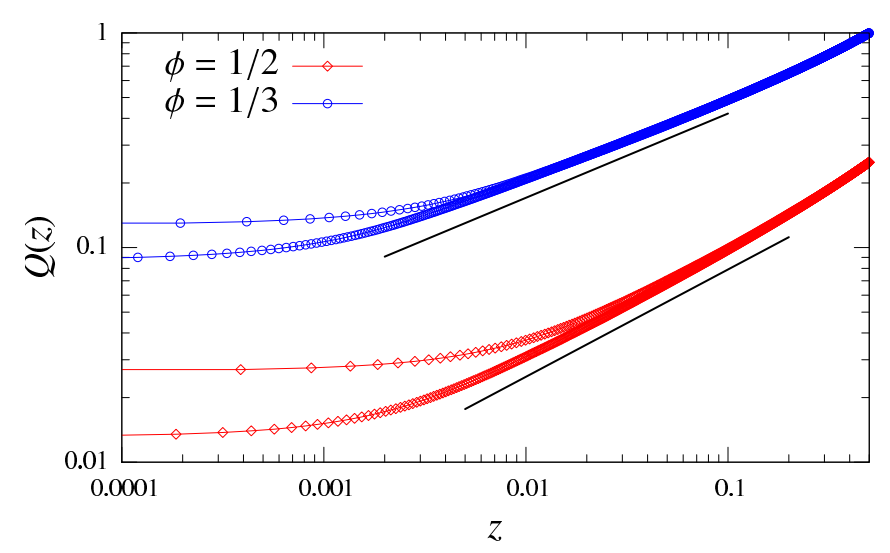

FIG. 3: Behavior of $Q(z)$ close to $z=0$ for fBm processes. For $H=2 / 3(\phi=1 / 2)$ the size of the box is $L=50,200$; for $H=3 / 4(\phi=1 / 3)$ the size of the box is $L=100,300$. The continuum limit is reached when $L \rightarrow \infty$. The expected slopes are reported as solid lines. Data have been shifted to make visualization easier.

holds also for the fractional Brownian motion (fBm), i.e., a self-affine Gaussian process defined by the following autocorrelation function

$$
\left\langle X\left(t_{1}\right) X\left(t_{2}\right)\right\rangle=\frac{1}{2}\left(t_{1}^{2 H}+t_{2}^{2 H}-\left|t_{1}-t_{2}\right|^{2 H}\right),
$$

with $0<H<1$ [13, 14]. In [7, we have proposed fBm as a natural candidate for describing the time evolution of the translocation coordinate. For this process, the persistence exponent is known, $\theta=1-H$ [14], so that $\phi=(1-H) / H$. An expedient algorithm for generating $\mathrm{fBm}$ paths is provided in 15 . The probability $Q(z)$ can be numerically computed as follows. Given a realization of the process starting from the origin, we record its minimum and maximum values for increasing time; the process is halted when $X_{\max }-X_{\min } \geq L$. If the last updated quantity is $X_{\min }$, the contribution to $Q(x, L)$ is 0 for $x \in\left(0, L-X_{\max }\right)$ and 1 for $x \in\left(L-X_{\max }, L\right)$. In the opposite case, the contribution is 0 for $x \in\left(0,-X_{\min }\right)$ and 1 othewise. All simulations are performed by averaging over $10^{6}$ samples. Fig. 3 shows the agreement between numerical simulations and predicted scaling of $Q(z)$ for different values of the parameter $H$.

Disordered potential. We next consider the stochastic motion of a single particle diffusing in a potential $V(X)$, starting at the initial position $x$. The dynamics is governed by the Langevin equation $\dot{X}(t)=f[X(t)]+\eta(t)$, where $X(0)=x$ and $f(X)=-d V(X) / d X$ is the force and $\eta(t)$ is a Gaussian white noise with $\langle\eta(t)\rangle=0$ and $\left\langle\eta(t) \eta\left(t^{\prime}\right)\right\rangle=\delta\left(t-t^{\prime}\right)$. To compute $Q(x, L)$, the first step is to write a differential equation satisfied by $Q(x, L)$, taking the initial position $x$ as a variable and keeping the box size $L$ fixed. Let us consider a small time interval $[0, \Delta t]$ at the beginning of the process. In this time interval, the particle moves from its initial position $x$ to a new position 


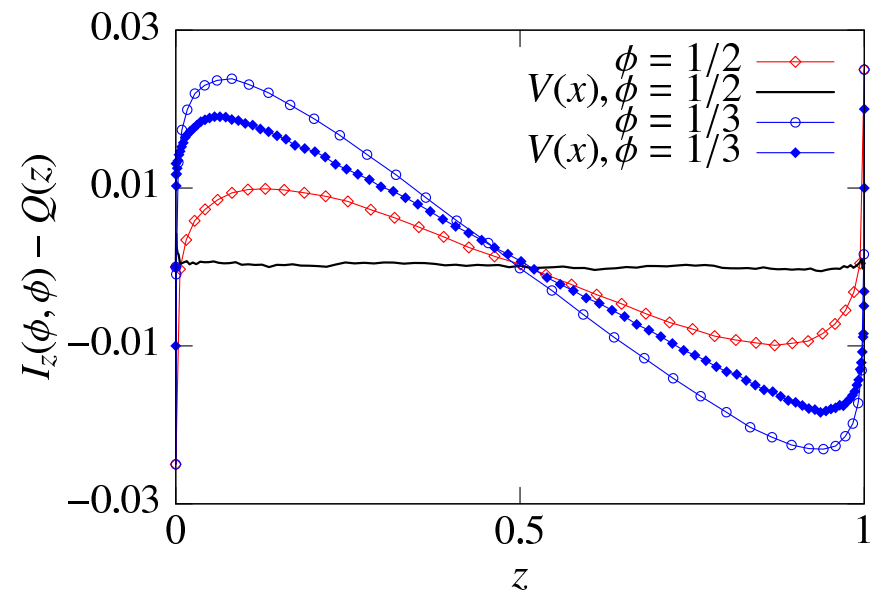

FIG. 4: The difference between Eq. (8) and simulated $Q(z)$. For fBm processes: $H=2 / 3(\phi=1 / 2)$ with box size $L=200$, and $H=3 / 4(\phi=1 / 3)$, with box size $L=300$. For $\mathrm{fBm}$ disordered potentials: $H_{V}=2 / 3(\phi=1 / 3)$, with box size $L=10^{4}$. For comparison, we display also the Sinai model $H_{V}=1 / 2(\phi=1 / 2)$, with box size $L=10^{4}$.

$x+\Delta x$ at time $\Delta t$, where $\Delta x=f(x) \Delta t+\eta(0) \Delta t, \eta(0)$ being the noise variable that kicks in at time 0 . Since the process is Markovian, the subsequent evolution does not know about the interval $[0, \Delta t]$, hence one gets

$$
Q(x, L)=\langle Q(x+f(x) \Delta t+\eta(0) \Delta t, L)\rangle,
$$

where \langle\rangle denotes the average over the initial noise $\eta(0)$. Expanding the rhs of Eq. (2) as a Taylor series in powers of $\Delta t$, using $\langle\eta(0)\rangle=0$ and $\left\langle\eta^{2}(0)\right\rangle=1 / \Delta t$ (delta correlated noise), yields an ordinary differential equation, $\frac{1}{2} Q^{\prime \prime}(x)+f(x) Q^{\prime}(x)=0$. Solving with boundary conditions $Q(0, L)=0$ and $Q(L, L)=1$ gives the exact result

$$
Q(x, L)=\frac{\int_{0}^{x} e^{2 V\left(x^{\prime}\right)} d x^{\prime}}{\int_{0}^{L} e^{2 V\left(x^{\prime}\right)} d x^{\prime}},
$$

valid for arbitrary potential $V(X)$. Note that for a potential-free particle, i.e., $V(X)=0$ in Eq. (3), we recover the Brownian result, $Q(x, L)=x / L$.

Taking derivative with respect to $x$ gives

$$
p_{\text {eq }}(x, L)=\frac{\partial}{\partial x} Q(x, L)=\frac{e^{2 V(x)}}{\int_{0}^{L} e^{2 V\left(x^{\prime}\right)} d x^{\prime}},
$$

which can be interpreted as the equilibrium probability density of the particle to be at $x$ in presence of a potential $-V(X)$. When $V(X)$ is a realization of a disordered potential, it is natural to introduce $\overline{Q(x, L)}$, the disorderaveraged hitting probability. An example where we can determine $\overline{Q(x, L)}$ explicitly is the classical Sinai model, i.e., when the potential $V(X)$ is a trajectory of a Brownian motion in space, $V(X) \sim X^{1 / 2}[16$. For this model the $\overline{p_{\text {eq }}(x, L)}$ can be computed exactly [17, 18]

$$
\overline{p_{\text {eq }}(x, L)}=\frac{1}{\pi} \frac{1}{\sqrt{x(L-x)}} .
$$

Thus, $\overline{Q(x, L)}=Q(z=x / L)$ again satisfies the generic scaling with a scaling function form with

$$
Q(z)=\frac{2}{\pi} \arcsin (\sqrt{z}) .
$$

Note that close to the origin $Q(z) \sim z^{\phi}$ with $\phi=1 / 2$. On the other hand, it is well known that in the Sinai potential the particle evolves very slowly with time, $X \sim \ln ^{2}(t)$, showing a self-affine scaling in the variable $T=\log t$, with $H=2$. For this model, it is also known that the survival probability decays as $1 / \log t$, i.e., $T^{-\theta}$, with $\theta=$ 1 19, 20]. Thus, $\theta / H=1 / 2=\phi$, in accordance with our general scaling prediction.

We next consider a generic self-affine potential, $V(X) \sim X^{H_{V}}$ (with $V(0)=0$ ), the Sinai potential being a special case with $H_{V}=1 / 2$. We show that $\overline{p_{\text {eq }}(x, L)}$ for such a potential is related to the probability density of the location $x_{m}$ of the maximum of the potential $V(X)$ over $X \in[0, L]$. We rewrite Eq. (4) as $p_{\text {eq }}(x, L)=\left[\int_{0}^{L} e^{2\left(V\left(x^{\prime}\right)-V(x)\right)} d x^{\prime}\right]^{-1}$, rescale variables $x^{\prime} \rightarrow x^{\prime} L$ and $x \rightarrow x L$ and use the self-affine property $V(x L)=L^{H_{V}} V(x)$ to obtain $p_{\text {eq }}(x, L)=$ $\left[\int_{0}^{1} e^{2 L^{H} V\left(V\left(x^{\prime}\right)-V(x)\right)} d x^{\prime}\right]^{-1}$. For large $L$, using a steepest decent method, we immediately see that, for each realization of the disorder potential $V(X), p_{\mathrm{eq}}(x, L) \simeq \delta\left(x-x_{m}\right)$ where $x_{m}$ denotes the position where $V(X)$ is maximum. This observation has two immediate consequences: (i) By integrating over $x$, we get, for each realization, $Q(x, L) \simeq \theta\left(x-x_{m}\right)$. This means that, for any given realization, if the starting position $x$ is to the left (right) of the location $x_{m}$ of the maximum, $Q(x, L) \simeq 0$ (respectively $Q(x, L) \rightarrow 1$ ) indicating that the particle exits the box through 0 (through $L$ ) as depicted in Fig. 2(right). (ii) By taking average over the disorder, we get

$$
\overline{p_{\text {eq }}(x, L)} \simeq p_{m}(x, L)
$$

where $p_{m}(x, L)$ is the probability density that the maximum of the potential $V(X)$ over $[0, L]$ is located at $x$. For example, for the Sinai case, one knows from Lévy's arcsine law [8] that $p_{m}(x, L)=1 / \pi \sqrt{x(L-x)}$. Thus, in this case the relation (7) is verified by the exact result (5). However, the relation (7) is more general and holds for arbitrary self-affine potential. We remark here that physically the relation (7) reflects the fact that in a self-affine potential $-V(X)$ in which the particle is at equilirium, the limit $L \rightarrow \infty$ limit is equivalent to the zero temperature $T \rightarrow 0$ limit forcing the particle to the minimuum of the potential $-V(x)$ or equivalently to the maximum $x_{m}$ of $V(x)$.

A useful consequence of (7) is that it allows us to relate the persistence or the survival probability of a particle moving in a disordered self-affine potential to the statistical properties of the potential $V(X)$ itself. The disordered potential $V(X) \sim X^{H_{V}}$ (we assume $V(0)=0$ ) can itself be regarded as a stochastic process with the 
space coordinate $X$ playing the role of 'time'. So, the probability that $V(X)$ stays below (or above) the level $X=0$ up to a distance $L$ decays, for large $L$, as $L^{-\theta_{V}}$ where $\theta_{V}$ is the spatial persistence exponent [21] of $V(X)$. For example, for the Sinai potential (brownian motion in space), $\theta_{V}=1 / 2$. The pair of exponents $\left(H_{V}, \theta_{V}\right)$ associated with the potential can now be related to the corresponding exponents associated with the temporal motion of the particle in this potential. By Arrhenius' law for the activated dynamics, the time required for particle diffusing in $V(X)$ to overcome an energy barrier scales as $t \sim e^{V(X)}$. Using $V(X) \sim X^{H_{V}}$, one deduces that $X \sim T^{1 / H_{V}}$ where $T=\log (t)$. Thus the particle motion $X(T) \sim T^{H}$ is a self-affine process as a function of $T=\log (t)$, with a Hurst exponent $H=1 / H_{V}$. Next, we note that $p_{m}(x, L)$, the probability that the maximum of $V(X)$ occurs at $x$, coincides, when $x \rightarrow 0$, with the probability that $V(X)$ stays below 0 up to a distance $L$, hence $p_{m}(x \rightarrow 0, L) \propto L^{-\theta_{V}}$. On the other hand, based on our general argument, we expect that $\overline{Q(x, L)} \sim(x / L)^{\phi}$ when $x \rightarrow 0$, where $\phi=\theta / H$. this means that $\overline{p_{\text {eq }}(x, L)} \propto x^{\phi-1} / L^{\phi}$. Note that here $\theta$ is the persistence exponent associated with the temporal motion of the particle, i.e., the survival probability of the particle up to time $T=\log (t)$ decays as $\sim T^{-\theta}$. Matching powers of $L$ from both sides of 7 provides the desired relation between temporal and spatial exponents $\theta=\theta_{V} H=\theta_{V} / H_{V}$. For instance, in the Sinai model, using $\theta_{V}=1 / 2, H_{V}=1 / 2$ we get and $\theta=1$, in agreement with the exact result [19, 20]. If one considers a potential $V^{\prime \prime}(X)=\xi(X)$ where $\xi(X)$ is a white noise in space, it is self-affine with $H_{V}=3 / 2$. The exponent $\theta_{V}=1 / 4$ is known exactly [26]. Thus we predict that for this poential, the survival probabaility up to time $t$ will decay as $\sim(\log t)^{-\theta}$ with $\theta=1 / 6$.

Super-universality of $Q(z)$. For some non-Brownian stochastic self-affine processes, the full function $Q(z)$ is known. For instance, Lévy Flights are Markovian superdiffusive processes whose increments obey a Lévy stable (symmetric) law of index $0<\mu \leq 2$. The Hurst exponent is $H=1 / \mu$. By virtue of the Sparre Andersen theorem [22], the persistence exponent is $\theta=1 / 2$, independent of $\mu$. Hence, $\phi=\theta / H=\mu / 2$ (see also [23, 24]). The full function $Q(z)$ for Lévy Flights has been computed 25] and can be recast in an elegant form

$$
Q(z)=I_{z}(\phi, \phi)=\frac{\Gamma(2 \phi)}{\Gamma^{2}(\phi)} \int_{0}^{z}[u(1-u)]^{\phi-1} d u
$$

i.e., a regularized incomplete Beta function containing a single parameter $\phi=\mu / 2$. Clearly, $Q(z) \sim z^{\phi}$ as $z \rightarrow 0$ in agreement with our prediction. The formulae for Brownian motion (with $\phi=1$ ) $Q(z)=z$ and for the Sinai model $(\phi=1 / 2)$ in (6) can also be expressed as (8). Moreover, the distribution of the maxima for a symmetric Lévy Flight process is given by (5), by virtue of the Sparre Andersen theorem [22]. Hence, we expect the hitting probability (8) to apply also to particles diffusing in a Lévy Flight disordered potential, with $\phi=1 / 2$. Finally, $Q(z)$ is known also for the Random Acceleration model, a non-Markovian process that is defined by $d^{2} X / d t^{2}=\eta(t)$, with $\eta(t)$ as before. The motion starts at $X(0)=x$, with initial velocity $v(0)=0$, and is superdiffusive, with $X \sim t^{3 / 2}$, i.e., $H=3 / 2$. Its firstpassage properties have been widely studied [26]. The persistence exponent is $\theta=1 / 4$, so that $\phi=\theta / H=1 / 6$. Bicout and Burkhardt 27] also computed the full exit probability $Q(x, L)$. One can again recast this formula in the same super-universal form (8) with $\phi=1 / 6$.

Based on these special cases, it may be tempting to conjecture that the full function $Q(z)$ for arbitrary anomalous doffusion processes has the super-universal form (8), the only information about the process enters this formula through a single exponent $\phi$. However, this turns out not to be the case, and we are able to show notable counterexamples. In Fig. 4, we compute the hitting probability for fBm self-affine processes and for particles diffusing in $\mathrm{fBm}$ disordered potentials, and display the numerical difference with respect to formula (8), with the appropriate exponent $\phi$. We find that in neither case $Q(z)$ can be described by the super-universal form (8).

* Electronic address: andrea.zoia@cea.fr

[1] A.Yu. Grosberg et al., Phys. Rev. Lett. 96, 228105 (2006).

[2] A. Meller et al. Phys. Rev. Lett. 86, 3435 (2001).

[3] D.K. Lubensky, D.R. Nelson, Biophys. J. 77, 1824 (1999).

[4] A.J. Storm et al., Nano Lett. 5, 1193 (2005).

[5] J. Chuang, Y. Kantor, M. Kardar, Phys. Rev. E 65, 011802 (2001).

[6] D. Panja, G.T. Barkema, R.C. Ball, J. Phys.: Condens. Matter 19, 432202 (2007).

[7] A. Zoia, A. Rosso, S.N. Majumdar, Phys. Rev. Lett. 102, $120602(2009)$.

[8] W. Feller, An Introduction to Probability Theory and its Applications (New York, Wiley, 1968).

[9] S. Redner, A Guide to First-Passage Processes (Cambridge University Press, Cambridge, 2001).

[10] J.-P. Bouchaud, A. Georges, Phys. Rep. 195, 127 (1990).

[11] B. Berkowitz et al. Rev. Geophys. 44, RG2003 (2006).

[12] S.N. Majumdar, Curr. Sci. 77, 370 (1999).

[13] B.B. Mandelbrot, J.W. van Ness, SIAM Rev. 10, 422 (1968).

[14] J. Krug et al., Phys. Rev. E 56, 2702 (1997).

[15] T. Dieker, http://www2.isye.gatech.edu/ adieker3/

[16] Y.G. Sinai, Theory Probab. Appl. 27, 256 (1982).

[17] A. Comtet, C. Texier, in Supersymmetry and Integrable Models, Aratyn et al. (eds.), Lecture Notes in Physics 502, 313, Springer (1998). cond-mat:/9707313.

[18] K. Broderix, R. Kree, Europhys. Lett. 32, 343 (1995).

[19] A Comtet, D.S. Dean, J. Phys. A: Math. Gen. 318595 (1998).

[20] P. Le Doussal, D.S. Fisher, C. Monthus, Phys. Rev. E 
64, 066107 (2001).

[21] S.N. Majumdar, A.J. Bray, Phys. Rev. Lett. 86, 3700 (2001).

[22] E. Sparre Andersen, Math. Scand. 1, 263 (1953).

[23] G. Zumofen, J. Klafter, Phys. Rev. E 51, 2805 (1995).

[24] A. Zoia, A. Rosso, M. Kardar, Phys. Rev. E 76021116 (2007)

[25] H. Widom, Trans. Amer. Math. Soc. 98, 430 (1961);
R.M. Blumenthal, R.K. Getoor, D.B. Ray, Trans. Amer. Math. Soc. 99, 540 (1961).

[26] T.W. Burkhardt, J. Phys. A 26, L1157 (1993); Y.G. Sinai, Theor. Math. Phys. 90, 219 (1992).

[27] D.J. Bicout, T.W. Burkhardt, J. Phys. A 33, 6835 (2000). 